\title{
Research and Publishing in Oral and Maxillofacial Surgery
}

\author{
George Dimitroulis
}

\subsection{Introduction}

We have all been too quick to make up our minds and too slow to change them. In Oral and Maxillofacial Surgery, our opinions are slaves to our prior experience. Ignorant confidence is what defined early Surgery as there was a distinct lack of interest in proving and promoting what was effective. The "God complex" among early Surgeons meant that what they thought was true did not need scientific evidence. It is only in more recent times that Surgical practice has embraced evidence to guard against rumour, bias, misconceptions and misunderstandings. Indeed, just in the last few decades we have witnessed the gradual evolution of Surgery from eminence-based practice, the idea that senior experienced Surgeons held all the knowledge, to evidence-based practice, where fair tests are employed to compare one treatment against the other in order to find what works best. History has taught us that clinical research leads to reforms in the practice of Surgery while basic research leads to revolutions in Medicine.

Like in most other scientific disciplines, Surgical research is used to confirm facts, reaffirm the results of previous work, solve existing problems and ultimately develop new ideas of practical value. If you can understand more, you can make better decisions rather than blind guesses which is the hallmark of successful clinical practice. Judgements, predictions and plans are based on the latest available information and should be constantly updated in the light of new information derived from good research. The late British Economist John Maynard Keynes once said "when the facts change, I change my mind". So too, a good Surgeon must be open to new ideas and techniques which are essential to the progress and evolution of each surgical specialty, as change is only possible when key opinion leaders lead the charge.

G. Dimitroulis $(\bowtie)$

Maxillofacial Unit, St. Vincent's Hospital - University of

Melbourne, Melbourne, Australia
Essentially, the greatest discoveries in Surgery are those that force us to rethink our beliefs about human disorders and our role in managing it. Research builds our core knowledge, and the most useful knowledge is one that changes the behaviour and practice of Surgeons. The simple idea of washing your hands before handling patients met with immense resistance from the established medical community in the nineteenth century until the scientific evidence supporting microbial infection became too overwhelming to ignore. Knowledge increases your ability to predict the outcomes, and the knowledge of bacterial infections was one of the greatest breakthroughs in modern medicine that significantly reduced the mortality and morbidity of even the simplest of surgical procedures. Research has been pivotal to the success of surgery over the last two centuries and will continue to be an integral part of all future progress, especially in the rapidly expanding field of Oral and Maxillofacial Surgery.

Progress doesn't occur in a vacuum; it almost always builds on existing ideas with a series of incremental improvements. That is why most research is used to develop further knowledge on a topic by reaffirming the results, theories and problems of past work in the field. Occasionally, when trying to solve an existing problem, research may lead to the discovery of new problems, ideas and theories which makes the science of Surgery a dynamic process of knowledge acquisition that has no endpoint.

\subsection{The Science of Research}

The word Science comes from the Latin "scienta" which means knowledge or skill. Science is about new ideas and testing these in the most transparent way. What launched the Scientific revolution was the realization that we do not have the answers to their most important questions. To find the answers, experiments are what people devised when they weren't sure of the truth. Ignorance is the starting point of all 
science, and curiosity about the world around us is what drives science. The more that is unknown, the greater the opportunity to discover, and that is how research has become an essential tool of discovery.

The word Research is derived from an old French term referring to seeking or searching. Research is all about the ability to test, reproduce, quantify and falsify an idea before it can be fully accepted as scientific fact. In research we learn more from the unexpected results than from those we anticipate. Understanding the research process helps generate new research questions. The trick is to ensure the right methods are used to answer the right questions. For example, when we are seeking verification, we must ask ourselves "Did I build the system right?", while if we want to validate something, we then ask "Did I build the right system?".

Scientific research is a systematic way of explaining things by collecting evidence which make practical applications possible. The goal of research is not only to yield new knowledge but also to make us better understand existing issues or topics. There are three main forms of Research; Exploratory research helps to identify a problem, while Constructive research proposes solutions to the problem. The third is Empirical research which tests the feasibility of the solution using either qualitative or quantitative methods. Qualitative research collects data in the form of words, images and video which is largely confined to the social sciences but may also have applications in Surgical techniques. The difference between quantitative (numbers) and qualitative (descriptions) research is well summarized by the famous scientist, Albert Einstein, who once said that "Not everything that matters can be measured, and not everything that can be measured matters". The world cannot be understood without numbers, and equally, it cannot be understood with numbers alone.

In Surgery, quantitative research is most often used to establish the existence of causal relationships between variables by collecting and analysing numerical data. By relying on random sampling, the quantitative research method allows for experimental, correlational and descriptive (i.e. survey) results that are easy to summarize, compare and generalize using statistics to determine the relationship between variables. For example, if the research question is about best mandibular reconstruction following tumour resection, the patients may be randomly assigned to different groups, each representing a different mode of reconstruction. If this is not feasible, the researcher may collect data related to the patient's demographics and situational characteristics to statistically control for their influence on the degree of morbidity following their particular mandibular reconstruction. Often the intent of the surgical research is to generalize from the study participants to a larger population which will require the researcher to employ probability sampling to select the study participants.
Table 45.1 The scientific research method

a. Identifying problem through observation

b. Review the literature-look for gaps in knowledge

c. Specify aims/purpose of research

d. Determine specific question or hypothesis

e. Define the variables being measured

f. Choose method of data collection-avoid bias

g. Data analysis-choose your statistical method

h Data interpretation-keep an open mind

i. Test/revision of hypothesis

j. Conclusion/recommendations for surgical practice

k. Reporting/communicating/publishing findings

\subsection{The Scientific Research Method} (Table 45.1)

Scientific research follows a structured process. Successful research is only possible when asking the right questions, engaging in the right observations, running the right experiments and networking with the right people to elicit ideas and feedback. The core principle of science and research are precision and transparency_-being clear about your methods and honest with your results. Transparency is what gives science credibility. World-changing ideas and technology were built from a protracted process of trial and error. Experiments are designed to show what does work and what doesn't. It is by testing that we gain access to the feedback that drives progress. Formal research has a well-established pathway that follows the basic steps set out below.

\subsubsection{Observation}

Many people simply watch the world around them (on autopilot). Few people observe. An astute observer will see the problem only when they're not vested in the way things have been. Their ability to imagine how things should be is what drives change and progress. The hardest part of solving a problem is seeing it, and many surgeons blindly follow procedures that do more harm than good for the patient because they have failed to question the surgical technique they have been taught. In surgery, very few surgeons have ever asked the pivotal question- "Is there a better way?" Curiosity and an open mind is what drives progress, and unfortunately, most surgeons have achieved their chosen profession by not rocking the boat and adhering to conventional practices.

Many of the things we accept as objective truths are themselves assumptions based on uncertainty, even in the world of Surgery. No surgical concept or technique has an absolute and permanent value. Time, experience and better experimental tools give rise to new ideas that supplant the old ideas. In other words, all existing surgical ideas and techniques are sooner or later supplanted by new ideas and techniques which means surgery is a never ending marvel of evolution. 
Before embarking on any kind of research, the first step is to look for any idea or technique that has not been adequately explained or supported by evidence. When looking to a topic or issue as a potential subject for research, the hierarchy of deliberation begins with (1) "Where is the evidence?", (2) "How sound is the evidence?" and (3) "Has the evidence been properly interpreted?". Once you have selected the appropriate subject or topic you would like to explore, the next step is to find out what the literature has to say and look for gaps in the existing knowledge.

\subsubsection{Literature Review}

All the world's information is now at our fingertips, and to access scientific publications is a matter of a few keystrokes. Search engines like Google Scholar and PubMed have opened up a whole new digital world of scientific literature that even the largest University libraries could never hope to subscribe to. Access to the world's scientific literature has never been simpler than it is today, and subscriptions to University libraries also allow students digital access to a wide range of local and international journals.

Once the research topic has been selected, a thorough review of the literature is required to establish what is already known. It is best to begin with the most recent peer-reviewed articles on the topic which will often summarize the existing body of knowledge. Meta-analysis papers, especially those that follow the PRISMA guidelines, are becoming more common, and these papers can form the foundation of your research topic. Good journal papers will commonly highlight the gaps in our knowledge, the weakness in the current evidence available, and what further research is required that will conveniently point you in the right direction.

\subsubsection{Purpose of Research}

Once you have gathered all the pertinent information from the literature review, the next step is to crystalize in your own mind why you are embarking on this research. The flaws or holes in previous research are identified so that the gaps in the literature will provide justification for the new research being proposed. Look for clues in the literature where there may be a deficiency in the evidence surrounding published statements, particularly those that make generalizations that are not adequately backed by evidence. Indeed, many Cochrane reviews have consistently concluded that the vast majority of clinical papers fail to provide the level of evidence required to make any firm conclusions. The aim or purpose of the research is pivotal to the whole project as it dictates the methodology required to adequately address the aims. It is also the main driver in funding applications and recruiting study participants.

\subsubsection{Hypothesis}

Science grows with every new piece of evidence with the hypothesis as the compass that directs the inquiries. Scientific theories have to make testable predictions that can be validated in experiments. Science is not just about confirmation; it is also about falsification. If a theory cannot be tested, then it's logically impossible to ever falsify it. Karl Popper said "if it is not falsifiable, it's not scientific". If a theory cannot be tested with experiments, then it falls in the realm of religion or philosophy or pure speculation. Questions that generate the greatest emotional response are a great indicator of challenging the way things are.

The gap in the literature creates a research question which is otherwise referred to as the Hypothesis. The Hypothesis is a prediction which is set out as a statement that defines the relationship between two or more variables. In research, it is the hypothesis that is being tested as to whether it is true or false. If the hypothesis turns out to be consistent with the observations, then it is said to be supported rather than proven as it is still subject to further scrutiny. But if it is rejected, then support is claimed for an alternative hypothesis. The Null hypothesis is when there is no relationship between the variables being investigated.

\subsubsection{Define the Variables}

In the hypothesis statement, there will be two or more variables that will be the focus of the study. The study is designed to establish the relationship between the variables. However, before commencing the study, the variables must be clearly defined so that there is no doubt as to what each variable represents. Without clear definitions of the variables, studies cannot be repeated and hence impossible to validate.

\subsubsection{Data Collection}

What isn't measured can't be managed. Without data, we cannot make informed decisions. We gather data to collect information that becomes knowledge which is finally displayed as wisdom. Data has to be collected, processed and analysed for it to become information which can be used to make better decisions. Ultimately, the answer is only as good as the quality of the data fed into it.

Apart from field studies such as surveys and clinical audits, OMF Surgeons also conduct experiments that involve laboratory controlled conditions, often by recruiting the resources of other medical specialities such as pathology, microbiology, radiology, biochemistry, pharmacology and haematology. Data collection is easiest when data points can be quantified or represented with numerical figures. Even 
descriptive surveys can be quantified provided the responses can be listed on a sliding scale ranging from good to bad, high to low or positive to negative. A typical example is the visual analogue scale used by OMF Surgery researchers to quantify pain scales, chewing ability and quality of life outcomes before and after jaw surgery.

In Medicine, randomized trials where the patients and experimenters are blinded are least vulnerable to bias than observational studies, which are most prone to bias. Unfortunately, randomized trials are more expensive than observational studies and require a lot more manpower and resources to properly execute. In surgery, we most commonly rely on observational studies as the best evidence we have to go on for two reasons. Firstly, control groups with which to compare treatment outcomes, unlike a placebo sugar drug, are rare as it is ethically impossible to undertake sham operations. And secondly, it is practically impossible to blind surgeons and patients when it comes to evaluating surgical procedures. Evidence in the field of Oral and Maxillofacial Surgery therefore relies largely on observational studies derived from surveys and clinical audits of case series.

\subsubsection{Data Analysis}

Data science is about understanding the world by spotting patterns and predicting how one variable will affect another. While our instincts may give us a reasonable sense of how the world works, we need data to sharpen the picture as we can be easily blinded by our own experiences and prejudices.

Data shows us there is more to OMF Surgery than we think we see. Good data science can often demonstrate counterintuitive results-what you least expected to see. Selection biases are distortions of the results introduced either by the data collecting tools or by the method of data accumulation.

Statistics is a valuable tool that helps determine whether the results you have found are likely to be due to a chance rather than a true finding. Make sure the data is collected on an Excel spreadsheet, and work out what level of confidence you will accept as statistically significant which, in clinical research, is often the $95 \%$ confidence level (i.e. $P$ value $<0.05)$. In statistics, the smaller the sample, the lower the likelihood that it would mirror the broader population. Large samples yield more precise results, while small samples are more susceptible to extreme results. As sample sizes get larger, the statistical calculations from that sample get more precise. Small differences $(1 \%)$ between two populations being measured require bigger sample numbers than large differences $(25 \%)$ for the true value to emerge. This is called the power calculation which is an important measure to con- sider before embarking on any study. Statistics is a complex affair and beyond the scope of this chapter, so the services of an expert statistician may well be useful when it comes to crunching the data.

\subsubsection{Data Interpretation}

Information is interpreted by different people in different ways. People can look at the same set of facts and disagree. Data can be manipulated to support any argument. You don't always need a ton of data to find important insights. What you need is the right data. Numbers can be seductive. We can grow fixated with them, and in doing so, we can lose sight of more important considerations. When looking at data, it is not the raw crunching power you have that matters most, but what you do with it that is most important. Behind every statistic there is a certain set of assumptions and prejudices. Minds crave certainty, and when they don't find it, they impose it so we must keep an open mind when the data does not yield the results we expected to see.

\subsubsection{Test or Revision of Hypothesis}

Your judgement call about how the results support your hypothesis is essential to your conclusions. If the data does not support your hypothesis, then it is essential to state an alternate hypothesis that fits with what has been observed. The scientific method is tailor-made for cherry picking because of its hypotheses and caveats and refusal to embrace certainty. Even in Science, certainty still relies on the assumptions, interpretations and theories of researchers based on what they see. Nothing is definite, for doubt is an essential operating principle of science. This means that doubt still has a place in all scientific proofs, so that all evidence is provisional and not final. In other words, what you have ascertained in your research is not definite proof but rather tacit support for an idea.

\subsubsection{Conclusions and Recommendations}

The conclusion is a summary of the entire experiment or study that simply states the outcomes observed which must be clear, concise and to the point. Any ambiguity should be highlighted and recommendations made as to how future studies should be conducted to minimize the uncertainty. It may simply be a numbers game where more patients are required, or additional groups such as control patients which may provide a clearer outcome. For example, the effective- 
ness of Botox in Myofascial Pain (TMD) can never be properly evaluated if there are no control groups (i.e. that have normal saline injected) with which to compare.

The conclusion is the most sought after piece of information that others will first look at when reviewing your research. Although it sounds rather obvious, the conclusion(s) must be supported by the data; otherwise, the research makes no sense. The onus is on the researcher to make sure the conclusions also fulfil the goals and aims of the study. Recommendations based on the conclusions are essential in providing a practical guide to surgical practice. Research is what drives clinical advances in Surgery, and the change in behaviour of Surgeons can only be achieved with evidence.

\subsubsection{Reporting/Communicating/ Publishing Findings}

Research is useless if it is not shared with the outside world. All humanity is now connected by digital technology which has rendered distance, time and costs irrelevant to the distribution and exchange of ideas. The digital world has made recording, storage and dissemination of information, research and ideas virtually free with costless reproduction and instantaneous global distribution. Reporting and publishing research findings is described in detail in Sect. 5.

\subsection{Constraints in Surgical Research}

Cultures that fail to encourage questioning also fail to come up with new ideas. Great institutions should teach us how to ask hard questions and where to look for answers. Knowing all the answers does not distinguish someone's intelligencerather, the ability to ask the right questions and linking the unconnected is the mark of a true genius. Generally, it is considered a weakness and a sign of vulnerability for clinicians to appear unsure. Confidence in medicine is valued over uncertainty, but science is all about uncertainty which drives progress. If we knew it all, there would not be a need for research.

Nothing is as powerful as an idea whose time has come. New ideas are what drive behavioural change and innovation. A new behaviour needs social approval before others are likely to pick up the habit as their own. Only the curious, who are open to learning, have a much greater chance of creating a truly novel solution. Unfortunately, originality is not always embraced by the surgical profession who are generally conservative by nature.

It is much easier for Surgeons to accept familiar ideas than totally original ones because if the ideas are too original, then it may be too hard for the Surgeon to accept or understand. The goal is to push the envelope, not to tear the envelope. Radical ideas are best presented in a way that is less shocking and more appealing to mainstream surgical practice by planting the seeds of a simple idea before revealing the larger idea. If you want your ideas to be accepted by your peers, make your ideas more appealing by connecting it with other ideas that are already understood by your colleagues. Other constraints related to research involve ethics, funding and bias which are discussed below.

\subsubsection{Ethics}

There has been a push in recent years by institutions and clinical journals to have all animal and human research activities sanctioned by appropriate ethics review boards or panels attached to hospitals or Universities. Without ethics approval or clearance, it may be impossible to conduct even the simplest of clinical studies, including basic surveys. Some Journals now request a copy of the ethics clearance letter before the paper can even be put out to review.

Unlike pharmaceutical research, the level of evidence in clinical surgery is hampered by the fact that control subjects are often missing because you cannot ethically perform sham operations in humans. Therefore, when designing surgical experiments, consider whether animal, laboratory or cadaver studies may be useful instead. Otherwise, you are limited to clinical audits which provide useful information which may not be scientifically valid. For example, a hospital which boasts $100 \%$ survival of its patients achieves this by turning away the sickest patients. Or a hospital which has the worst outcomes may well be a tertiary referral centre that only treats the most complex of cases that other hospitals turn away. So, while clinical audits provide a snapshot of the Hospital Unit's activities, it tells us very little else as far as science, progress and innovation is concerned.

We must be suspicious of therapeutic claims that have not been properly tested. Equally, we must be even more suspicious of research results that cannot be replicated. Many people are driven by a deep human desire for recognition and affirmation of work well done. Sometimes it is possible to build an academic career by sounding clever, rather than being clever. Most academics chase large numbers of trivial publications instead of investing their energies in new frontiers. In other words, why search for something new when you can collect rents on everything that has already been done. Sadly, there are small numbers of academics and researchers who have built their careers on falsification and plagiarism. The desire for recognition and the need for promotion when combined with the pressure to publish can tip some academics, clinicians and scientists towards fraudulent activities where data is made up and text is plagiarized in 
order to churn out the maximum number of "scientific" papers. In science and surgery, reputation takes many years of hard dedication to build up and an instant to destroy when you're suspected of scientific fraud.

\subsubsection{Funding}

Scientists have an innate desire to innovate, share, collaborate and be recognized for it regardless of the financial incentives. Hence, good research requires funding from external sources. Unfortunately, the spirit of open scientific enquiry can sometimes be hijacked by the combination of self-interest and money, especially when industry offer to bankroll the study. Industry rely on profitable enterprises which grow shareholder value. Scientific support behind a product is perhaps the ultimate value-adding marketing tool, so industry are always looking out for research that shows positive outcomes related to their products. Furthermore, industry never leave anything to chance so they want control of scientific research through financial rewards to various research institutions. By directly funding research, industry have control over what is published (i.e. the positive results) and what stays buried in a locked cupboard (i.e. the negative results).

Therefore, the most respectable research is that which is funded by government or not-for-profit organizations such as Medical Societies and disease interest groups like the Heart Foundation or the Cancer Council and so on. Funding from non-industry groups are much more competitive as there is always a limited amount of money available for research so applications must be of high quality. Furthermore, the nonindustry funding bodies give free reign to the scientists on how the data is used and published. Industry funding, on the other hand, have simpler applications but more control of the data which remains their property. Most importantly, industry insist on the final veto on whether or not the results are published.

\subsubsection{Confirmation Bias}

Confirmation bias is one of the shortcomings of science because the human mind is bad at seeing things it did not expect to see and a bit too eager to see what it expected to see. It is basically seeing what you want to see and ignoring everything else. In essence, confirmation bias is when you filter reality through biases by eagerly accepting evidence that confirms what you believe and ignoring evidence that refutes or challenges what you believe. Once we adopt a particular hypothesis or interpretation, we find it difficult to see things any other way. People will accept any explanation as long as it fits with their own understanding of the facts.

\subsubsection{Cognitive Dissonance}

Cognitive dissonance is another impediment to scientific progress that affects many people. It is simply a feeling of discomfort that people experience when presented with information that is inconsistent with their beliefs. When we are confronted with evidence that challenges our deeply held beliefs, we are more likely to reframe the evidence than we are to alter our beliefs. In most cases, instead of acknowledging an error in judgement, people tend to reformulate their views in a way that justifies their old opinions. We simply invent new reasons, new justifications, or new explanations or ignore it altogether. Some people go to absurd lengths to justify their beliefs or judgement even when confronted with clear contrary evidence. The more committed we are to a certain opinion, the less likely we are to relinquish it, even when confronted with massive or overwhelming contradictory evidence. As an example, the introduction of microvascular venous couplers in microsurgery was developed by those who could see the obvious benefits of speed and patency of the anastomosis, and yet was resisted by those who felt the skill of micro-suturing tiny veins would be lost, regardless of the benefits of reduced thrombosis and improved flap survival.

\subsection{Publishing}

Research cannot thrive without publishing. All research projects are conceived with publication as the end game. The excessively competitive field of research creates a tendency to rush to conclusions and publish results that have not been properly validated. Valid conclusions can only be drawn from reproducible data sets because results that initially look promising aren't always repeatable. It is little wonder that remarkably few published breakthroughs have ever led to any useful treatments.

There are numerous media that researchers can use to propagate their experimental findings which are discussed below.

\subsubsection{Electronic Media}

Digital technology has not only transformed but has revolutionized the way we live. The merger of the personal computer and the Internet allowed networking to blossom on a massive scale, and so surgeons and scientist are not wholly reliant on print media to propagate their ideas. Websites like ResearchGate encourage scientists and clinicians to upload their research, both published and unpublished, onto a digital platform for all to see and to foster collaboration between research groups across the world. 
Digital media platforms like YouTube are fast becoming a quick, easy and virtually cost-free way of publishing your novel ideas and surgical techniques on the Internet for all to see. More and more Surgeons are bypassing the heavily fortified realm of print media for the highly accessible digital media which not only offers a global audience but also facilitates immediate upload of content with virtually no delay. The disadvantage is of course the lack of scrutiny which suggests the content has not been vetted by experts. Hence, the scientific value of the video post is virtually zero. While you may have a wide audience, it may not necessarily be the people you want to impress.

\subsubsection{Magazines}

Glossy magazines that specifically target general medical/ dental practitioners are supported by lots of industry advertising which is interspersed with clinical articles that have not been peer-reviewed, but rather submitted on the invitation of the magazine editor. The articles, which often have more clinical pictures than text, are written by clinicians and academics as a general interest piece that provides the reader with an update of what is current clinical practice that is aimed at the non-specialist practicing clinician. Authors are generally paid for their contributions. These articles are of little value to the specialist trainee or clinician wanting to find out more about their own specialty.

\subsubsection{Textbooks}

Textbooks contain information that is current practice and accepted by the profession. Unfortunately, by the time a textbook is commissioned, written, published and finally released, the information is often about 2 years out of date. Therefore, the strength of textbooks is in the basic principle of surgical practice, and the weakness lies in the distinct lack of new information. Digital technology is gradually changing the need for printed textbooks as students are gravitating to knowledge that is presented in discrete digital packages with hyperlinks that cross-reference important concepts similar to "Wikipedia". While university and hospital libraries still purchase textbooks for student consumption, today's students are finding the expense of textbooks prohibitive and so seek other means of accessing surgical information and knowledge through the Internet, which is often up to date as long as they know what to look for and are able to critically evaluate the credibility of the information source.

\subsubsection{Peer-Reviewed Journals}

There are over 1 million academic papers published every year in over 24,000 academic journals where gaps in our knowledge are discussed and new experiments are conducted that might resolve these gaps. Unfortunately, published scientific papers tend to be biased towards reporting positive results. Negative results do not make headlines so they are rarely published. Publication in a journal is not a mark of truth but merely that the research has passed a certain standard that warrants entering the formal literature and further discussion.

Scientific journals sell scholarship back to the same universities whose scientists had produced, written, peer reviewed and edited largely for free. Hence the cost of producing scientific journals is kept as low as possible in order to facilitate as wide a distribution as possible. Unlike magazines, peer-reviewed journals with few exceptions keep industry advertising to a minimum as they are supposed to be impartial to avoid conflict of interest when reporting studies that may conflict with the interests of a big advertiser.

Peer-reviewed journals are the platform we use to announce new discoveries, to comment on or criticize the discovery of others and to synthesize and seek to build consensus about what is known. In Oral and Maxillofacial Surgery, case reports and technical notes account for about one-third of journal publications, while less than one in ten are randomized control (Level 2 evidence) or non-randomized control (Level 3 evidence) studies (Table 45.2). Because of the difficulty in performing high-level randomized control trials in surgery, Surgeons are more accepting of lower-level evidence which is based on observation. Indeed, it is unlikely to extract any high-level evidence research from surgical departments who rely on surgical trainees for their research output.

Table 45.2 Levels of evidence (Oxford Centre for Evidence-Based Medicine)

Level 1: Randomized control trials (RCT)

High-quality, properly powered and conducted studies with reduced bias when double blinded and multicentred

Level 2: Cohort clinical studies

Prospective, comparative clinical study without randomization

Level 3: Case-controlled studies

Retrospective clinical study

Level 4: Case series studies

Cross-sectional study (clinical audit) with or without intervention

Level 5: Case-based reasoning

Includes case reports, expert opinions and bench research

Note: The strength of systematic reviews and meta-analysis depends on the evidence levels of the papers being reviewed. If all the papers are Level 3 evidence, the systematic review will also be Level 3 evidence 
Table 45.3 Factors to consider in the design of a clinical study

1. Where is the study being conducted? (field, hospital, clinic, laboratory, etc.)

2. Are there ethical issues? (animal or human studies)

3. Define your study groups or variables being investigated

4. Selection criteria (inclusion/exclusion)

5. Are there matched controls?

6. Describe the surgical or technical details

7. Method of data collection (randomized or blinded)

8. Method of data handling (statistical method)

Table 45.4 Criteria for a good surgical manuscript

1. Short, succinct title

2. Original ideas with practical or pragmatic applications

3. Clear purpose/aim(s)

4. Good grammar-clear and concise wording/short sentences

5. Methods described in adequate detail to allow independent verification

6. Results set out in clearly labelled tables, graphs, figures, photos, diagrams

7. Focused discussion with balanced literature that both support and refute results

8. The most recent references that adequately support discussion

9. Conclusion supported by the results of the study

Table 45.5 Anatomy of a poor surgical manuscript
1. Long, convoluted and ambiguous title
2. Wild, unusual, unethical or dangerous ideas of little practical value
3. No clear aims or purpose stated
4. Plagiarism and duplication (study previously published elsewhere under different title)
5. Poor grammar-incorrect wording/long convoluted sentences/ emotive language
6. Methods inadequately or poorly described that cannot be repeated
7. No data, tables, figures, graphs, photos or diagrams provided
8. Convoluted discussion without clear direction and biased to supportive literature
9. Old or antiquated references
10. Conclusion not supported by the results of the study

All journals subscribe to the IMRD layout which means all submissions must have an Introduction, Methods, Results and Discussion sections. The introduction builds the case for why you pursued the line of research and the final sentence is always the statement of the aim(s) of your study. The Methods section describes how the study was performed in terms of setting (e.g. Hospital or Laboratory), subjects (i.e. animals or humans), recruitment (e.g. inclusion/exclusion criteria), data collection (e.g. Surveys, measurements), variables being compared (experimental vs. control) and data analysis (statistics used) (Table 45.3). The Results section simply states the summary of the outcomes as depicted in the tables and graphs, while the Discussion critically analyses and compares the results in light of what has already been previously published on the topic. The discussion must include references that both agree and disagree with your findings so that a balanced argument can be presented which will add weight to the study.
Table 45.6 Guidelines for formatting papers for publication
1. Randomized control studies:

2. Observational studies:

3. Systematic reviews:

4. Case series:

5. Case reports: www.consort-statement.org www.strobe-statement.org www.prisma-statement.org www.processguideline.com www.scareguideline.com
The final paragraph in the discussion is a summary of the research results with mention of any practical clinical applications that may arise from the study.

Authors must keep in mind that originality and a clear message are essential in getting their papers published in the highly competitive world of scientific journal publishing (Table 45.4). The high impact factor journals generally attract articles with high evidence levels so it is imperative to select a journal that caters to the subject and evidence levels of your particular study to avoid rejection and delays in publication of your paper. Original contributions that add new information to the existing body of knowledge are more likely to be considered for publication. However, wild or fanciful ideas are unlikely to garner support from journal editors who are looking for papers that lend respectability to their journal.

Unlike works of fiction, good scientific communication is based on clear and concise wording with short titles and tightly controlled sentences that describe complex ideas in the simplest language possible. Poor grammar, emotive language and long-winded descriptive wording must be avoided so that the ideas being conveyed are not buried in a convoluted tangle of discourse (Table 45.5). When evaluating the importance and relevance of published articles, surgeons and scientists look for the facts, not fancy prose.

To improve the acceptability of your paper for publication in a peer-reviewed journal, there are general guidelines available online that help you set out your paper in a format that is recognized and accepted throughout the world (Table 45.6). By following the guidelines, you improve the chances of your paper being accepted for publication. For instance, if you want to submit an observational case cohort study, then the "STROBE" statement will guide you in how to properly format your paper. If it is a case report then it is worth checking out the "SCARE" guidelines. Alternatively, if you want to undertake a systematic review of a topic, the "PRISMA" statement is essential.

\subsection{Conclusion}

In Surgery there is no greater accomplishment than being the first to successfully implement a successful treatment that changes the history of medicine. However, the person with the first idea is usually not the one recognized by history. Credit often goes to the person who convinces the world, not to the one with the original idea, because the credibility of all new ideas requires convincing evidence. If you make a claim 
about something, you provide the evidence, or at least a reference to the evidence that backs your claim. Research is the essential tool that builds the evidence which provides a better understanding of how and why things worked or failed. Good surgical practice must be based on evidence.

Surgical innovation is a constant work in progress because any new ideas or techniques are quickly supplanted by an even better ideas and techniques. Gaps in the literature are what fuel research, and the journey of discovery is paved with potholes that need to be filled. To paraphrase the great scientist Sir Isaac Newton, if you want to see further than anyone else has seen before, you need to stand on the shoulders of giants. Your voyage of discovery begins once you have secured your footing on the shoulders of the surgeons and scientists who have gone before you, so you can see the path they have built for you to follow.

\section{Further Reading}

Dimitroulis G. EDITORIAL - Getting published in peer-reviewed journals. Int J Oral Maxillofac Surg. 2011;40:1342-5.

Dimitroulis G. EDITORIAL - Research - why bother? Int J Oral Maxillofac Surg. 2011;40:1346.

Hardicre J. An overview of research ethics and learning from the past. Br J Nurs. 2014;23:483-6.

Knottnerus JA, Tugwell P. EDITORIAL - Evidence-based medicine: achievements and prospects. J Clin Epidemiol. 2017;84:1-2.

Lau SL, Samman N. Levels of evidence and journal impact factor in Oral \& Maxillofacial Surgery. Int J Oral Maxillofac Surg. 2007;36:1-5.

Mohan M, Shetty D, Shetty T, Pandyak K. Rising from plagiarising. J Maxillofac Oral Surg. 2015;14:538-40.

Sollaci LB, Pereira MG. The introduction, methods, results, discussion (IMRAD) structure: a fifty year survey. J Med Libr Assoc. 2004;92:364-7.

Szklo M. Impact factor: good reasons for concern. Epidemiology. 2008;19:369.

Open Access This chapter is licensed under the terms of the Creative Commons Attribution 4.0 International License (http://creativecommons. org/licenses/by/4.0/), which permits use, sharing, adaptation, distribution and reproduction in any medium or format, as long as you give appropriate credit to the original author(s) and the source, provide a link to the Creative Commons license and indicate if changes were made.

The images or other third party material in this chapter are included in the chapter's Creative Commons license, unless indicated otherwise in a credit line to the material. If material is not included in the chapter's Creative Commons license and your intended use is not permitted by statutory regulation or exceeds the permitted use, you will need to obtain permission directly from the copyright holder. 